\title{
Non-Equilibrium Phase Transition in Rapidly Expanding Matter
}

\author{
I.N. Mishustin \\ The Niels Bohr Institute, Blegdamsvej 17, DK-2100 Copenhagen Ø, Denmark; \\ The Kurchatov Institute, Russian Research Center, Moscow 123182, Russia
}

\begin{abstract}
Non-equilibrium features of a first order phase transition from the quarkgluon plasma to a hadronic gas in relativistic heavy-ion collisions are discussed. It is demonstrated that strong collective expansion may lead to the fragmentation of the plasma phase into droplets surrounded by undersaturated hadronic gas. Subsequent hadronization of droplets will generate strong non-statistical fluctuations in the hadron rapidity distribution in individual events. The strongest fluctuations are expected in the vicinity of the phase transition threshold.
\end{abstract}

PACS numbers: 12.38.Mh; 12.39.Fe; 25.75.-q

The main goal of present and future experiments with relativistic heavy ions is to produce and study in the laboratory a new form of strongly interacting matter, the Quark-Gluon Plasma (QGP). Due to the confinement of color charges, only colorless hadronic final states can be observed experimentally. Therefore, QGP properties can be studied only indirectly through the final hadron distributions or by penetrating electromagnetic probes. Many QGP signatures have been proposed in the recent years, particularly ones which assume an equilibrium phase transition between QGP and hadronic gas.

The phase structure of QCD is not yet fully understood. Reliable lattice calculations exist only for baryon-free matter where they predict a second order phase transition or crossover at $T_{c} \approx 160 \mathrm{MeV}$. Recent calculations using different models 표 reveal the possibility of a first order phase transition at large baryon chemical potentials and moderate temperatures. The predicted phase diagram in the $(T, \mu)$ plane contains a first order transition line (below called the critical line) terminated at $T \approx 120 \mathrm{MeV}$ by a (tri)critical point [2,3]. Possible 
signatures of this point in heavy-ion collisions are discussed in Ref. [5]. Under certain nonequilibrium conditions, a first order transition is also predicted for baryon-free matter [6].

A striking feature of relativistic heavy-ion collisions, confirmed in many experiments (see e.g. [7]), is a very strong collective expansion of matter. The applicability of equilibrium concepts for describing phase transitions under such conditions becomes questionable. The goal of this paper is to demonstrate that non-equilibrium phase transitions in rapidly expanding matter can lead to interesting phenomena which, in a certain sense, can be even easier to observe.

To make the discussion below more concrete, I adopt a picture of the chiral phase transition for which the mean chiral field $\Phi=(\sigma, \pi)$ serves as an order parameter. It is assumed that the theory respects chiral symmetry, which is spontaneously broken in the vacuum where $\sigma=f_{\pi}, \pi=0$. The effective thermodynamic potential $\Omega(T, \mu ; \Phi)$ depends, besides $\Phi$, on temperature $T$ and baryon chemical potential $\mu$. Since explicit symmetry breaking terms are supposed to be small, to a good approximation $\Omega$ is a function of $\Phi^{2}=\sigma^{2}+\pi^{2}$.

The schematic behaviour of $\Omega(T, \mu ; \Phi)$ as a function of the order parameter field $\sigma$ at $\pi=0$ is shown in Fig. 1. The curves from bottom to top correspond to different stages of the isentropic expansion of homogeneous matter. Each curve represents a certain point on the $(T, \mu)$ trajectory. The minima of $\Omega$ correspond to the stable or metastable states of matter under the condition of thermodynamical equilibrium, where the pressure is $P=-\Omega_{\min }$. The figure is based on the calculations within the linear $\sigma$-model with constituent quarks [1], which predicts a rather weak first order phase transition. A similar structure of $\Omega(T, \mu ; \Phi)$ but, possibly, with a stronger phase transition is predicted by the NJL model [2] and by the random matrix model [3]. The discussion below is quite general.

Assume that at some early stage of the reaction thermal (but not necessarily chemical) equilibrium is established and partonic matter is in a "high energy density" phase Q. This state corresponds to the absolute minimum of $\Omega$ with the order parameter close to zero, $\sigma \approx 0, \pi \approx 0$, and chiral symmetry restored (curve 1 ). Due to a very high internal pressure, Q matter will expand and cool down. At some stage a metastable minimum appears in 
$\Omega$ at a finite value of $\sigma$ corresponding to a "low energy density" phase $H$, in which chiral symmetry is spontaneously broken. At some later time, the critical line in the $(T, \mu)$ plane is crossed where the $\mathrm{Q}$ and $\mathrm{H}$ minima have equal depths, i.e. $P_{\mathrm{H}}=P_{\mathrm{Q}}$ (curve 2). At later times the $\mathrm{H}$ phase becomes more favourable (curve 3), but the two phases are still separated by the potential barrier. If the expansion of the $\mathrm{Q}$ phase continues until the barrier vanishes (curve 4), the system will find itself in an absolutely unstable state at a maximum of the thermodynamic potential. Therefore, it will freely roll down into the lower energy state corresponding to the $\mathrm{H}$ phase. This situation is known as a spinodal instability.

According to the standard theory of homogeneous nucleation [8], supercritical bubbles of the $\mathrm{H}$ phase appear only below the critical line. Under condition of thermal equilibrium between the two phases, the supercritical bubbles can only grow through the conversion of new portions of the $\mathrm{Q}$ matter into the $\mathrm{H}$ phase on the bubble boundary. The bubble growth is then limited by a small viscosity of the $\mathrm{Q}$ phase resulting in a slow dissipation of the latent heat [8]. Therefore, a certain degree of supercooling is needed in order to convert a significant fraction of the $\mathrm{Q}$ matter into the $\mathrm{H}$ phase in the form of nucleation bubbles [8,9].

In rapidly expanding matter the nucleation picture might be very different. Let us consider first an isotropically expanding system with the collective velocity field which follows the Hubble law locally, $v(r)=H \cdot r$. The Hubble "constant" $H$ may in general be a function of time, e.g. $H \sim 1 / t$. Obviously, the same velocity field is seen from any local rest frame comoving with the matter. Suppose that a bubble of the $\mathrm{H}$ phase has formed in the expanding $\mathrm{Q}$ matter because of a statistical fluctuation. The change in thermodynamic potential of the system can be decomposed into three parts,

$$
\Delta \Omega=\Delta \Omega_{\text {bulk }}+\Delta \Omega_{\text {surf }}+\Delta \Omega_{\text {kin }}
$$

In the thin-wall approximation the bulk and surface terms are expressed through the bubble radius $R$ in a standard way,

$$
\Delta \Omega_{\text {bulk }}=-\frac{4 \pi}{3} R^{3}\left(P_{\mathrm{H}}-P_{\mathrm{Q}}\right), \quad \Delta \Omega_{\text {surf }}=4 \pi R^{2} \gamma
$$


where $P_{\mathrm{H}}$ and $P_{\mathrm{Q}}$ are the pressures of the bulk $\mathrm{H}$ and $\mathrm{Q}$ phases, $\gamma$ is the effective surface tension. The last term in Eq. (四) accounts for the change in the local kinetic energy of expanding matter. In the same approximation it can be evaluated as

$$
\Delta \Omega_{k i n}=\frac{1}{2} \int_{0}^{R} 4 \pi r^{2} d r \mathcal{E}(r) v^{2}(r) \approx-\frac{2 \pi}{5} R^{5} \Delta \mathcal{E} H^{2},
$$

where $\Delta \mathcal{E} \equiv \mathcal{E}_{\mathrm{Q}}-\mathcal{E}_{\mathrm{H}}$ is the difference of energy (more exactly, enthalpy) densities of the two bulk phases. It is evident that this term is negative, since the dense $\mathrm{Q}$ phase is replaced in the bubble by the dilute $\mathrm{H}$ phase (typically, $\mathcal{E}_{\mathrm{Q}} \gg \mathcal{E}_{\mathrm{H}}$ ). Thus, the bubble formation is favoured by the collective expansion. Moreover, the nucleation can start now even above the critical line, when $P_{\mathrm{H}}<P_{\mathrm{Q}}$, and the standard theory would predict no growing bubbles. In principle, the phase separation can start as early as the metastable $H$ state appears in the thermodynamic potential, and a stable interface between the phases may exist.

Using Eqs. (21) and (3) one can determine the critical bubble radius $R_{c}$, corresponding to the top of the potential barrier in $\Delta \Omega(R)$. The condition $\partial_{R} \Delta \Omega=0$ leads to a cubic equation for $R_{c}$. When $H \rightarrow 0$ the kinetic term vanishes and this equation gives a standard Laplace formula for the critical bubble [8]. However for realistic parameters (see below) the kinetic term dominates. In particular, in the vicinity of the critical line, when $P_{\mathrm{H}} \approx P_{\mathrm{Q}}$, one can consider the bulk term perturbatively. Then one obtains

$$
R_{c}=\left(\frac{4 \gamma}{\Delta \mathcal{E} H^{2}}\right)^{1 / 3}\left[1-\frac{P_{\mathrm{H}}-P_{\mathrm{Q}}}{3\left(2 \gamma^{2} \Delta \mathcal{E} H^{2}\right)^{1 / 3}}\right] .
$$

The bubbles with $R>R_{c}$ will expand further while those with $R<R_{c}$ will eventually shrink. From the above consideration one should conclude that in a rapidly expanding system an appreciable amount of nucleation bubbles and even empty cavities will be created already above the critical line.

The bubble formation and growth will also continue below the critical line. Previously formed bubbles will now grow faster due to increasing pressure difference, $P_{\mathrm{H}}-P_{\mathrm{Q}}>0$, between the two phases. It is most likely that the conversion of $\mathrm{Q}$ matter on the bubble boundary is not fast enough to saturate the $\mathrm{H}$ phase. Therefore, a fast expansion may lead 
to a deeper cooling of the $\mathrm{H}$ phase inside the bubbles compared to the surrounding $\mathrm{Q}$ matter. Strictly speaking, such a system cannot be characterized by the unique temperature. At some stage $\mathrm{H}$ bubbles will percolate, and the topology of the system will change. Now isolated regions of the $\mathrm{Q}$ phase (Q droplets) will be surrounded by the undersaturated vapor of the $\mathrm{H}$ phase.

Standard thermodynamical concepts cannot be used in this non-equilibrium situation. However, the characteristic droplet size can be estimated by applying the energy balance consideration first proposed by Grady [10,11] in the study of dynamical fragmentation. The idea is that the fragmentation of expanding matter is a local process minimizing the sum of surface and kinetic (dilational) energies per fragment volume. The predictions of this simple model are in reasonable agreement with molecular dynamics simulations [11,12 and experimental data on dynamical fragmentation of fluids and solids (see e.g. [10,11, 13]). As shown in Ref. [14], this prescription works fairly well also for multifragmentation of expanding nuclei, where the standard statistical approach fails.

Let us imagine an expanding spherical Q droplet embedded in the background of the dilute $\mathrm{H}$ phase. In the droplet rest frame the change of thermodynamic potential compared to the uniform $\mathrm{H}$ phase is given by the same expression (11) but with indexes $\mathrm{H}$ and $\mathrm{Q}$ interchanged. The kinetic term is positive now. According to the Grady's prescription, the characteristic droplet radius, $R^{*}$, can be determined by minimizing

$$
\left(\frac{\Delta \Omega}{V}\right)_{\text {droplet }}=-\left(P_{\mathrm{Q}}-P_{\mathrm{H}}\right)+\frac{3 \gamma}{R}+\frac{3}{10} \Delta \mathcal{E} H^{2} R^{2} .
$$

It is worth noting that the collective kinetic energy term acts here as an effective long-range potential, similar to the Coulomb potential in nuclei. Since the bulk term does not depend on $R$ the minimization condition constitutes the balance between the collective kinetic energy and interface energy. This leads to an optimum droplet radius

$$
R^{*}=\left(\frac{5 \gamma}{\Delta \mathcal{E} H^{2}}\right)^{1 / 3}
$$

It should be noticed that this radius is expressed through the same combination of model parameters as the critical bubble radius at $P_{\mathrm{H}} \approx P_{\mathrm{Q}}$, Eq. (舟), but with a slightly bigger 
numerical coefficient. This suggests that in vicinity of the critical line the $\mathrm{H}$ and $\mathrm{Q}$ phases occupy roughly equal fractions of the total volume. Fast expansion (large $H$ ) may lead to very small droplets. This mixed state of matter is far from thermodynamical equilibrium because of the excessive interfacial energy and undersaturation of the $\mathrm{H}$ phase. One can say that the metastable $\mathrm{Q}$ matter is torn apart by a mechanical strain associated with the collective expansion. This has a direct analogy with the fragmentation of pressurized fluids leaving nozzles [12,13]. In a similar way, splashed water forms droplets which have nothing to do with the equilibrium liquid-gas phase transition.

At ultrarelativistic collision energies associated with RHIC and LHC experiments, the expansion will be very anisotropic, with its strongest component along the beam direction. Applying the same consideration for the anisotropic flow, one can see that the characteristic size of inhomogeneities in each direction is determined by the respective Hubble constant acting in this direction. Thus, the resulting structures will have smaller size in the direction of stronger flow. Therefore, in the case of strong one-dimensional expansion the inhomogeneities associated with the phase separation will rearrange themselves into pancake-like slabs of Q matter layered by the dilute $\mathrm{H}$ phase. The characteristic width of the slab is given by Eq. (6) with a slightly different geometrical factor. At a later stage the slabs will further fragment into smaller droplets. In general one should expect the emergence of complicated multi-fractal structures.

The driving force for expansion is the pressure gradient, $\nabla P=c_{s}^{2} \nabla \mathcal{E}$, which depends on the sound velocity in the matter, $c_{s}$. In the vicinity of the critical line one may expect a "soft point" [15,16] where $c_{s}$ is smallest and the ability of matter to generate the collective expansion is minimal (small $H$ ). If the initial state of the $\mathrm{Q}$ phase is close to this point, the primordial bubbles or droplets will be biggest. Increasing initial pressure will result in a faster expansion and smaller droplets. For numerical estimates I choose two values of the Hubble constant: $H^{-1}=20 \mathrm{fm} / \mathrm{c}$ to represent the slow expansion from the soft point [15] and $H^{-1}=6 \mathrm{fm} / \mathrm{c}$ for the fast expansion [9].

One should also specify two other parameters, $\gamma$ and $\Delta \mathcal{E}$. The surface tension $\gamma$ is a 
subject of debate at present. Lattice simulations indicate that at the critical point it could be as small as a few $\mathrm{MeV} / \mathrm{fm}^{2}$. However, for the non-equilibrium situation discussed here the values of $10-20 \mathrm{MeV} / \mathrm{fm}^{2}$, which follow from effective chiral models, should be more appropriate. As a compromise, the value $\gamma=10 \mathrm{MeV} / \mathrm{fm}^{2}$ is used below. It is clear that $\Delta \mathcal{E}$ should be close to the latent heat of the transition, i.e. about $0.5 \div 1 \mathrm{GeV} / \mathrm{fm}^{3}$. One can also estimate $\Delta \mathcal{E}$ by realizing that nucleons and heavy mesons are the smallest droplets of the $\mathrm{Q}$ phase. For estimates I take $\Delta \mathcal{E}=0.5 \mathrm{GeV} / \mathrm{fm}^{3}$, i.e. the energy density inside the nucleon. Substituting these values in Eq. (6) one gets $R^{*}=3.4 \mathrm{fm}$ for $H^{-1}=20 \mathrm{fm} / \mathrm{c}$ and $R^{*}=1.5 \mathrm{fm}$ for $H^{-1}=6 \mathrm{fm} / \mathrm{c}$.

In the lowest-order approximation the characteristic droplet mass is $M^{*} \approx \Delta \mathcal{E} V$. For spherical and slab-like droplets one gets respectively

$$
M_{s p}^{*} \approx \frac{20 \pi}{3} \frac{\gamma}{H^{2}}, \quad M_{s l}^{*} \approx 2 S(\Delta \mathcal{E})^{2 / 3}\left(\frac{3 \gamma}{H^{2}}\right)^{1 / 3}
$$

where $S$ is the slab transverse area. It is interesting to note that in this approximation $M_{s p}^{*}$ is independent of $\Delta \mathcal{E}$. For the two values of $R^{*}$ given above $M_{s p}^{*}$ is $\sim 100 \mathrm{GeV}$ and $\sim 10$ $\mathrm{GeV}$, respectively. The slab-like structures would have even larger mass, since $S$ could be of order of the transverse system size. Using the minimum information principle one can show [11,14 that the distribution of droplets should follow an exponential law, $\exp \left(-\frac{M}{M^{*}}\right)$. Therefore, with $1 \%$ probability one can find droplets as heavy as $5 M^{*}$.

After separation, the droplets recede from each other according to the global Hubble expansion, predominantly along the beam direction. Therefore, their center-of-mass rapidities are in one-to-one correspondence with their spatial positions. Presumably they will be distributed more or less evenly between the target and projectile rapidities. At this late stage it is unlikely that the thermodynamical equilibrium will be re-established between the $\mathrm{Q}$ and $\mathrm{H}$ phases or within the $\mathrm{H}$ phase alone. If this were to happen, the final $\mathrm{H}$ phase would be uniform, and thus there would be no traces of the mixed phase in the final state.

The final fate of individual droplets depends on their sizes and on details of the equation of state. Due to the counter-acting pressure of the $\mathrm{H}$ phase and additional Laplace pressure 
the initial expansion will slow down. In smaller droplets the expansion and cooling may even reverse to the contraction and reheating. The conversion of $\mathrm{Q}$ droplets into the $\mathrm{H}$ phase may proceed through formation of a deflagration front [16] or evaporation of hadrons from the surface [17]. Bigger droplets may expand further until they enter the region of spinodal instability. As shown in Ref. [18], the characteristic time of the "rolling down" process is rather short, $\sim 1 \mathrm{fm} / \mathrm{c}$, so that the $\mathrm{Q}$ droplets will be converted rapidly into the $\mathrm{H}$ phase. The energy released in this process can be transferred partly into the collective oscillations of the $(\sigma, \pi)$ fields. Numerical simulations [19,20] show that these oscillations persist for a long time and give rise to soft pion radiation. One should also expect the formation of Disoriented Chiral Condensates (DCC) in the voids between droplets.

Since rescatterings in the dilute $\mathrm{H}$ phase are rare, most hadrons produced from individual droplets will go directly into detectors. One may guess that the number of produced hadrons is proportional to the droplet mass. Each droplet will give a bump in the hadron rapidity distribution around its center-of-mass rapidity [18]. If emitted particles have a Boltzmann spectrum, the width of the bump will be $\delta y \sim 2 \sqrt{T / m}$, where $T$ is the droplet temperature and $m$ is the particle mass. At $T \sim 100 \mathrm{MeV}$ this gives $\delta y \approx 2$ for pions and $\delta y \approx 1$ for nucleons. These spectra might be slightly modified by the residual expansion of droplets and their transverse motion. The resulting rapidity distribution in a single event will be a superposition of contributions from different droplets, and therefore it will exhibit strong non-statistical fluctuations. The fluctuations will be more pronounced if primordial droplets are big, as expected in the vicinity of the soft point. If droplets as heavy as $100 \mathrm{GeV}$ are formed, each of them will produce up to $\sim 300$ pions within a narrow rapidity interval, $\delta y \sim 1$. Such bumps can be easily resolved and analyzed. Critical fluctuations of similar nature were discussed recently in Ref. [21].

Some unusual events produced by high-energy cosmic nuclei have been already seen by the JACEE collaboration [22]. Unfortunately, they are very few and it is difficult to draw definite conclusions by analyzing them. We should be prepared to see thousands of such events in the future RHIC and LHC experiments. It is clear that the nontrivial 
structure of the hadronic spectra will be washed out to a great extent when averaging over many events. Therefore, more sophisticated methods of the event sample analysis should be used. The simplest one is to search for non-statistical fluctuations in the hadron multiplicity distributions measured in a fixed rapidity bin [23]. One can also study the correlation of multiplicities in neighbouring rapidity bins, bump-bump correlations etc. Such standard methods as intermittency and commulant moments [21], wavelet transforms [24], HBT interferometry 25] can also be useful. All these studies should be done at different collision energies to identify the phase transition threshold. The predicted dependence on the Hubble constant and the geometry of reaction, Eq. (7), can be checked in collisions with different ion masses and impact parameters.

One should bear in mind two important points. First, if the expansion trajectory goes close to the (tri)critical point, both $\gamma$ and $\delta \mathcal{E}$ will tend to zero and the critical fluctuations will be less pronounced. Second, if a first order phase transition is possible only in the baryon-rich matter, then the Q droplets should have much higher baryon density than the hadronic phase [3]. In this case one should expect strong non-statistical fluctuations in the distribution of the net baryon charge.

In conclusion, simple arguments based on the homogeneous nucleation picture and the energy balance consideration demonstrate that a first order phase transition in rapidly expanding matter should proceed through a non-equilibrium stage when the metastable phase fragments into droplets. If QCD matter undergoes a first order phase transition, it will manifest itself in relativistic heavy-ion collisions by the formation of droplets of quark-gluon plasma. The primordial droplets should be biggest in the vicinity of the soft point where the expansion is slowest. The fragmentation of plasma might be accompanied by the formation of multiple DCC domains and enhanced soft-pion radiation. Subsequent hadronization of QGP droplets will lead to large non-statistical fluctuations in the hadron rapidity spectra in individual events. All these novel phenomena can only be detected through dedicated event-by-event analysis of experimental data.

The author is grateful to J.P. Bondorf and A.D. Jackson for many fruitful discussions. 
I thank Agnes Mocsy for preparing the figure. Discussions with G. Carter, D. Diakonov, J.J. Gaardhoje, L. McLerran, R. Mattiello, L.M. Satarov and E.V. Shuryak are greatly appreciated. I thank the Niels Bohr Institute for kind hospitality and financial support.

[1] L.P. Csernai, I.N. Mishustin and A. Mocsy, Heavy Ion Physics, 3, 151 (1996); A. Mocsy, M.Sc. thesis, University of Bergen, 1996.

[2] J. Berges and K. Rajagopal, Nucl. Phys. B (in press); hep-ph/9804233.

[3] M.A. Halasz, A.D. Jackson, R.E. Shrock, M.A. Stephanov and J.J.M. Verbarshot, Phys. Rev. D58, 096007 (1998).

[4] G. Carter and D. Diakonov, Nucl. Phys. A (in press); hep-ph/9807219.

[5] M. Stephanov, K. Rajagopal and E. Shuryak, Phys. Rev. Lett. 81, 4816 (1998).

[6] I.N. Mishustin, L.M. Satarov, H. Stoecker and W. Greiner, hep-ph/9812319.

[7] P. Braun-Münzinger and J. Stachel, Nucl. Phys. A638, 3c (1998).

[8] L.P. Csernai, J.I. Kapusta, Phys. Rev. Lett. 69, 737 (1992); Phys. Rev. D46, 1379 (1992).

[9] E.E. Zabrodin, L.V. Bravina, L.P. Csernai, H. Stoecker and W. Greiner, Phys. Lett. B423, 373 (1998).

[10] D.E. Grady, J. Appl. Phys. 53(1), 322 (1981).

[11] B.L. Holian and D.E. Grady, Phys. Rev. Lett. 60, 1355 (1988).

[12] J.A. Blink and W.G. Hoover, Phys. Rev. A32, 1027 (1985).

[13] H.Buchenau et al., J. Chem. Phys. 92, 6875 (1990).

[14] I.N. Mishustin, Nucl. Phys. A630, 111c (1998). 
[15] C.M. Huang, E.V. Shuryak, Phys. Rev. Lett. 75, 4003 (1995); Phys. Rev. C57, 1891 (1998).

[16] D. Rischke, M. Gyulassy, Nucl. Phys. A597, 701 (1996); A608, 479 (1996).

[17] M. Alford, K. Rajagopal and F. Wilczek, Phys. Lett. B422, 247 (1998).

[18] L.P. Csernai, I.N. Mishustin, Phys. Rev. Lett. 74, 5005 (1995).

[19] A. Abada, M. Birse, Phys. Rev. D55, 6885 (1987).

[20] I.N. Mishustin, O. Scavenius, Nucl. Phys. A638, 519c (1998).

[21] N.G. Antoniou, Nucl. Phys. B71, 307 (1999).

[22] T.H. Barnett et al., Phys. Rev. Lett. 50, 2062 (1983).

[23] M.J. Tannenbaum and E802 Collaboration, Phys. Rev. C52, 2663 (1995)

[24] N. Suzuki, M. Biyajima and A. Ohsawa, hep-ph/9503403.

[25] H. Heiselberg, A.D. Jackson, hep-ph/9809013. 


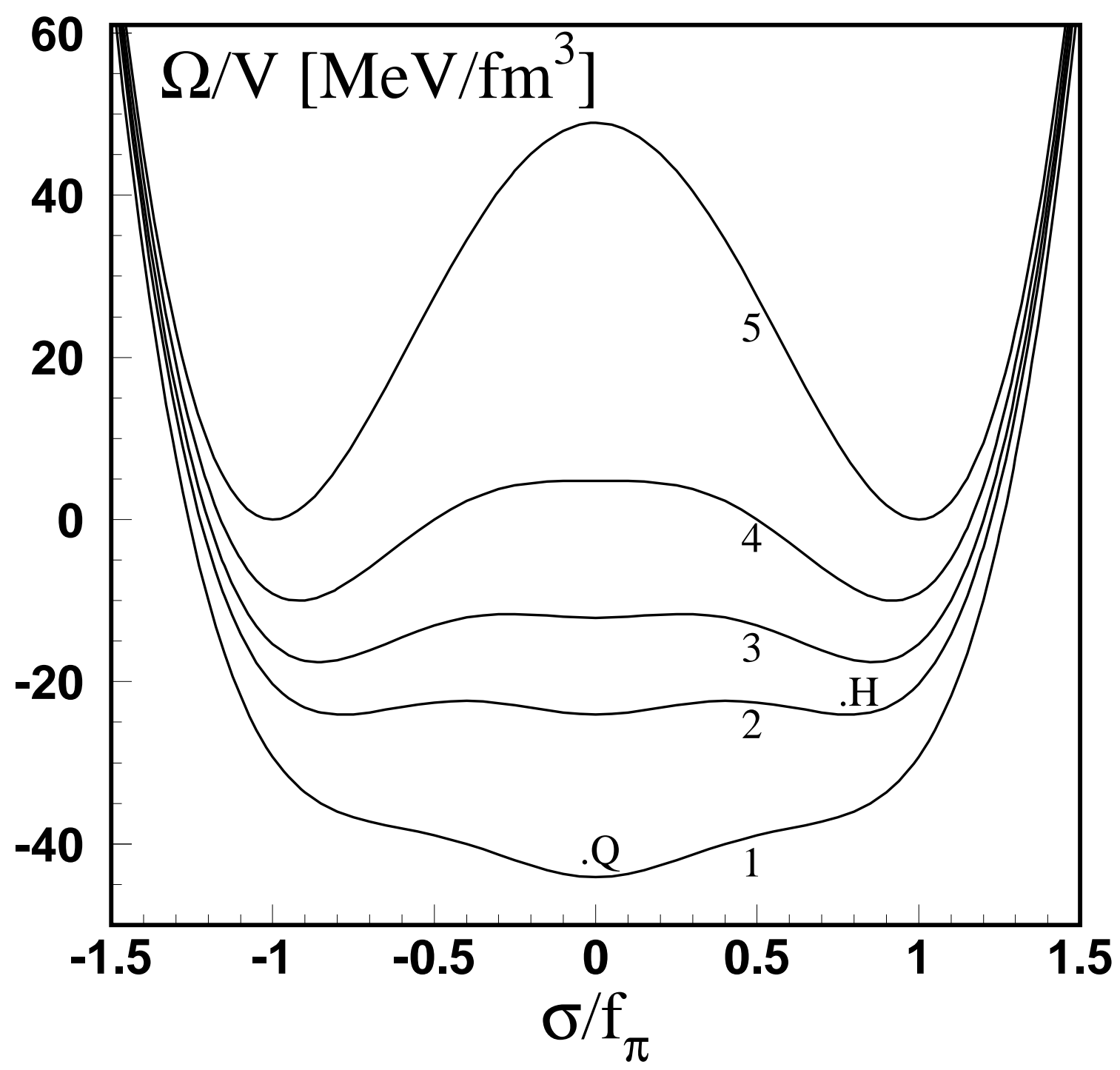

FIG. 1. Schematic view of the effective thermodynamic potential per volume $\Omega / V$ as a function of the order parameter field $\sigma$ at $\pi=0$, as predicted by the linear $\sigma$-model in the chiral limit $m_{\pi}=0$ [1]. The curves from bottom to top correspond to the different stages of the isentropic expansion of homogeneous matter starting from $T=100 \mathrm{MeV}$ and $\mu=750 \mathrm{MeV}$ (curve 1). The upper curve 5 is the vacuum potential. The other curves are discussed in the text. 